\title{
A Real Time and Interactive Web-Based Platform for Visualizing and Analyzing COVID-19 in Canada
}

\author{
Dan Liu ${ }^{1}$, Yuan $\mathrm{Du}^{2}$, Yasin Khadem Charvadeh ${ }^{1}$, Jingyu Cui ${ }^{1}$, Li-Pang Chen ${ }^{1}$, Gansen Deng ${ }^{1}$, Qihuang Zhang ${ }^{4}$, \\ Kaida $\mathrm{Cai}^{2}$, Joy $\mathrm{He}^{5}$, Wenqing $\mathrm{He}^{1,3}$, Grace $\mathrm{Y} . ~ \mathrm{Yi}^{1,2}$ \\ ${ }^{1}$ Department of Statistical and Actuarial Sciences, Western University, London, Ontario, Canada \\ 2 Department of Computer Science, Western University, London, Ontario, Canada \\ ${ }^{3}$ Department of Oncology, Western University, London, Ontario, Canada \\ ${ }^{4}$ Department of Statistics and Actuarial Science, University of Waterloo, Waterloo, Ontario, Canada \\ ${ }^{5}$ College of Natural Resources, University of California, Berkeley, Berkeley, California, United States \\ Correspondence: Wenqing He, Department of Statistical and Actuarial Sciences, Department of Oncology, Western \\ University, London, Ontario, N6A 3K7, Canada. E-mail: whe@ stats.uwo.ca
}

Received: June 8, 2020 Accepted: July 6, 2020 Online Published: July 28, 2020

doi:10.5539/ijsp.v9n5p23 URL: https://doi.org/10.5539/ijsp.v9n5p23

\begin{abstract}
In the midst of the global outbreak with over 300,000 worldwide death cases of COVID-19, Canada has reported 79,101 confirmed cases of the novel coronavirus (COVID-19) as of May 19, 2020, in which the severity differs from region to region. To provide a timely view and understanding of the evolving pandemic in Canada, we develop a real time interactive web-based platform which primarily includes data visualization and statistical analysis. The website highlights real time tracking of the development of COVID-19 with visualized graphs and forecasts future trends with applications of different statistical predictive models. By providing research-based statistical analysis, we are able to shed the light on the epidemiological characteristics of COVID-19. We also provide timely social news and preventive measures from the government on the website.
\end{abstract}

Keywords: Canada, COVID-19, data visualization, interactive web-based platform, statistical analysis

\section{Introduction}

Since the first case of COVID-19 was reported in Canada in January 2020, there have been a gradual increase of cases until the late March 2020 before the number of confirmed cases began to increase exponentially. As a result, measures for mitigating the spread of the novel coronavirus, such as states of emergency and social distancing have been declared one after another by the government in each province of Canada (https://www.canada.ca/en/public-health/services/diseases/2019-novel-coronavirus-infection.html?topic=tilelink).

Although those measures are critical in controlling the undetected transmissions of the virus, the seriousness of the evolving COVID-19 situation varies from region to region. These factors include testing capacity, distribution of age, hospital resources, and so on using the data from the Government of Canada, provincial governments and public sources. We develop a web-based interactive platform (https://covid-19-canada.uwo.ca/) that reports the real-time evolvement of COVID-19 in different regions of Canada by providing informative visualization from multiple angles and statistical analysis using different models. The focus is on Ontario, Alberta, British Columbia, and Quebec, the four provinces in Canada that have the most serious situations. Our objectives are to provide timely summarized displays of the development of COVID-19 in Canada to offer real time evidence-based findings and to create an interactive platform of up-to-date information on COVID-19 in Canada.

This platform was developed by the research group co-led by Grace Y. Yi and Wenqing He with the support from the Faculty of Science at Western University. The interactive and dynamic graphs on the site have been developed using Plotly in R (Sievert, 2020). It was officially launched in May 2020 and serves as the first platform in Canada providing comprehensive research-based statistical analysis with particular focus on COVID-19 situation in Canada. Among the sections on the site to be described below, DATA VISUALIZATION, STATISTICAL ANALYSIS, and RESEARCH MANUSCRIPTS are the spotlights as we believe that research-based statistical insights are critical in the fight against COVID-19. 


\section{Home}

First, we provide a clickable map on the homepage, as shown in Figure 1, which depicts real time COVID-19 situations for each province in Canada. The color on the map varies from province to province, showing different degrees of severity of COVID-19. This map vividly shows a brief summary of the key information, including confirmed/death/recovered/active/hospitalized/ICU cases, when readers hover the mouse over a particular province or territory. Furthermore, to help the public acquire timely and detailed analysis, we enable the map on the homepage to lead the readers to another page, DATA VISUALIZATION, that consists of analysis results when they click a province of interest.

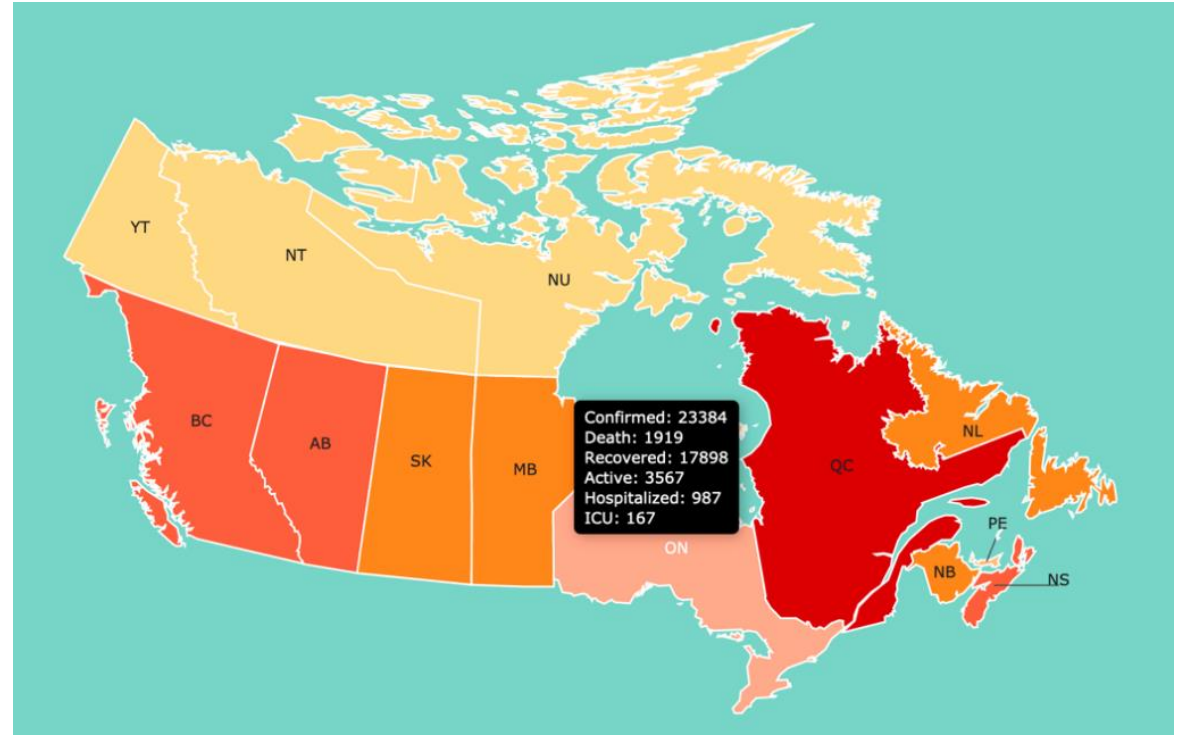

\section{Data Visualization}

Figure 1. Clickable map on the homepage

DATA VISUALIZATION proceeds to further track real time trends with visualized displays of the characteristics of COVID-19 such as the cumulative and daily cases, deaths, and recoveries at the province level, especially those who are the most severely infected. All the visualized graphs in this section are interactive and detailed information can be displayed. In addition, we provide zoomable heatmaps for readers to obtain a sense of the severity of the area at first glance when they are directed to DATA VISUALIZATION. The severity is evaluated based on the cumulative cases for the entire Canada heatmap and the active cases in a particular province for the provincial heatmap. An example of the heatmap in Ontario is shown in Figure 2. 


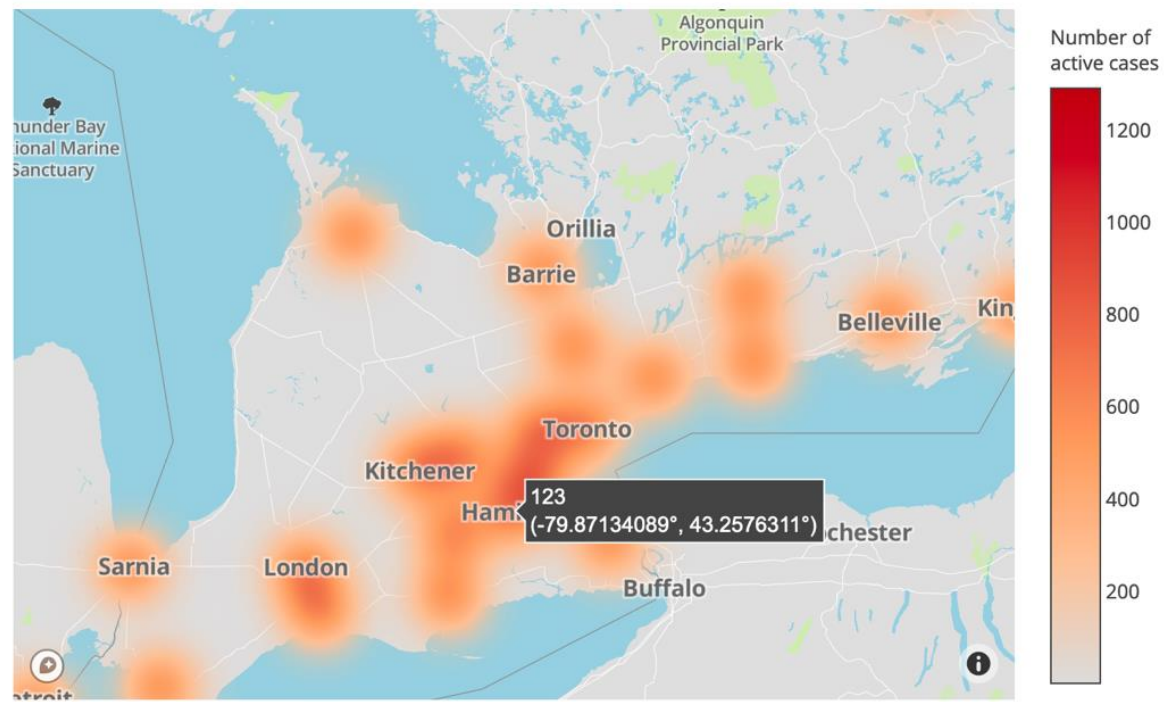

Figure 2. Heatmap in Ontario

Given the scant knowledge about COVID-19, we look at the data from various angles and describe the characteristics of COVID-19 in fuller and finer dimensions. We visualize the data at the province level by offering epidemiological assessment graphs such as epidemic curves of real-time cumulative/daily cases, proportions of patients' health status, and infection sources. Furthermore, we examine city-level proportions of cases, fatality/recovery rates in relation to both geographical and demographic features, which are not commonly evaluated in the public and government reports. To our amazement, some interesting discoveries can be uncovered from this. For instance, although male confirmed cases are less reported in Ontario, it turns out that males have a surprisingly higher fatality rate but a lower recovery rate than females across almost all the age groups, as shown in Figure 3. This pattern applies to Alberta, especially, for the age group of 40 years old or older, as shown in Figure 4. This revelation may help healthcare providers assess the situation cautiously.
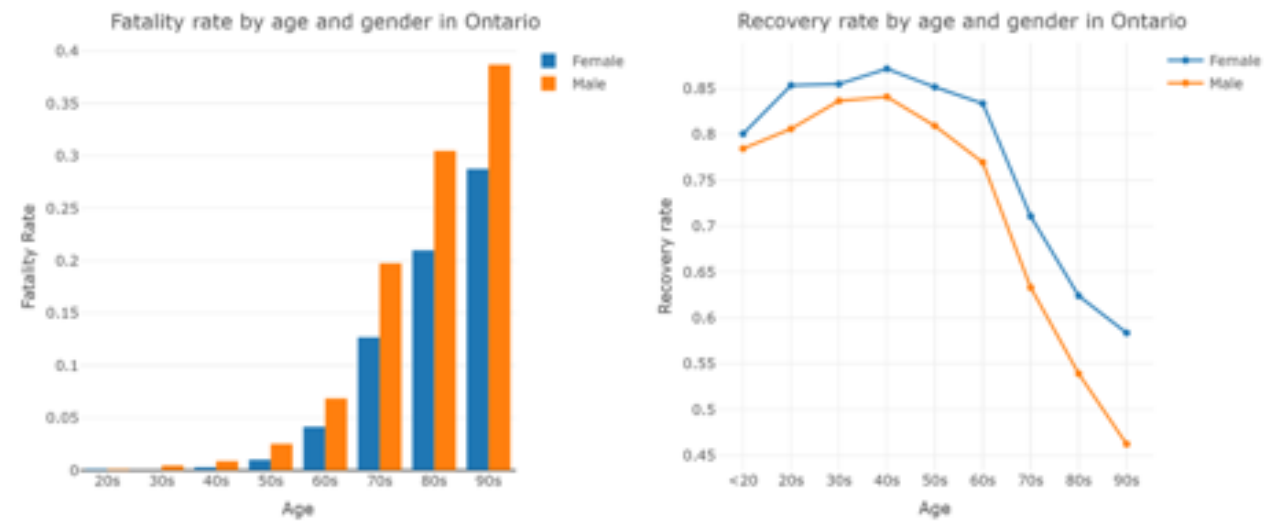

Figure 3. Fatality rate and recovery rate by age and gender in Ontario 

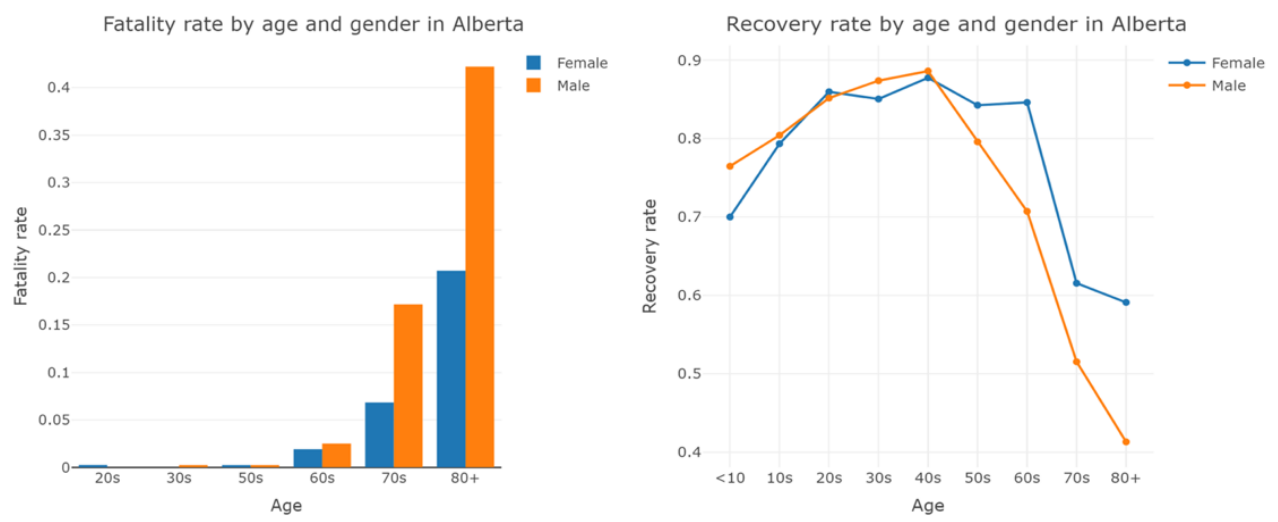

Figure 4. Fatality rate and recovery rate by age and gender in Alberta

In addition, we explore different representations of the same data to reveal more meaningful insights behind. Despite real-time cumulative case reporting, lab test rates and infection rates (infection cases per 1,000 people) in each province are also pictured on the site to show a more comprehensive view about the severity of COVID-19 in different provinces. While lab test numbers may commonly be reported, lab test rates at the province level, which measures the percentage of people in each province who have been tested for COVID-19, are rarely considered. In Figure 5, the infection rate reflects the percentage of confirmed cases in each province. As of May 19, Alberta ranks on the top in terms of lab test rate across Canada while its infection rate is about the same as in Ontario, this comparison may indicate the severity of COVID-19 in Alberta is less than that of Ontario.
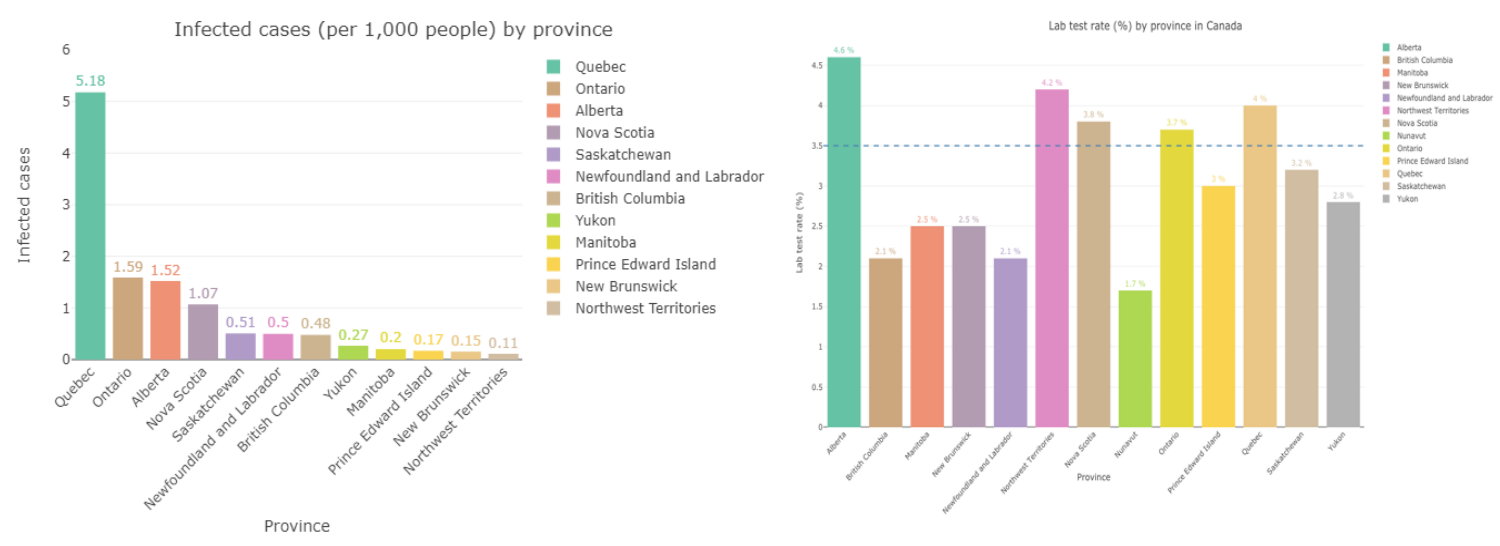

Figure 5. Infection rate (infection cases per 1,000 people) and lab test rate (\%) by province

\section{Statistical Analysis}

STATISTICAL ANALYSIS employs different statistical predictive models to regularly predict the development of COVID-19 in Canada in the next 7 days. At present, we consider two models for prediction, the SIR (susceptible-infected-resolved) model and the NN (neural network) method. The SIR model is known to be a compartmental model of infectious disease in epidemiology (Fanelli \& Piazza, 2020; Wang et al., 2020) while the NN method is a popular machine learning method that shows its advantage by its ability to approximate nonlinear function (Oancea \& Ciucu, 2014). Inspired by the currently exponential epidemic trend of COVID-19, we hope to compare how different methods may predict the trends in order to gain a broader picture under different conditions.

Despite the simple theoretical principles behind the SIR model, its implementation is based on strict assumptions that the infection rate needs to be constant over time with recovered and death information provided (Keeling \& Rohani, 2011). The validity of the SIR model can be significantly affected if any of the assumptions are violated. On the other hand, the NN method can be easily implemented but requires a suitable number of hidden layers and the number of nodes within them (Chatfield \& Xing, 2019; Patterson \& Gibson, 2017). Figures 6 and 7 forecast the future cumulative confirmed cases for Ontario, Alberta, British Columbia, and Quebec using the SIR model and NN method, respectively. 

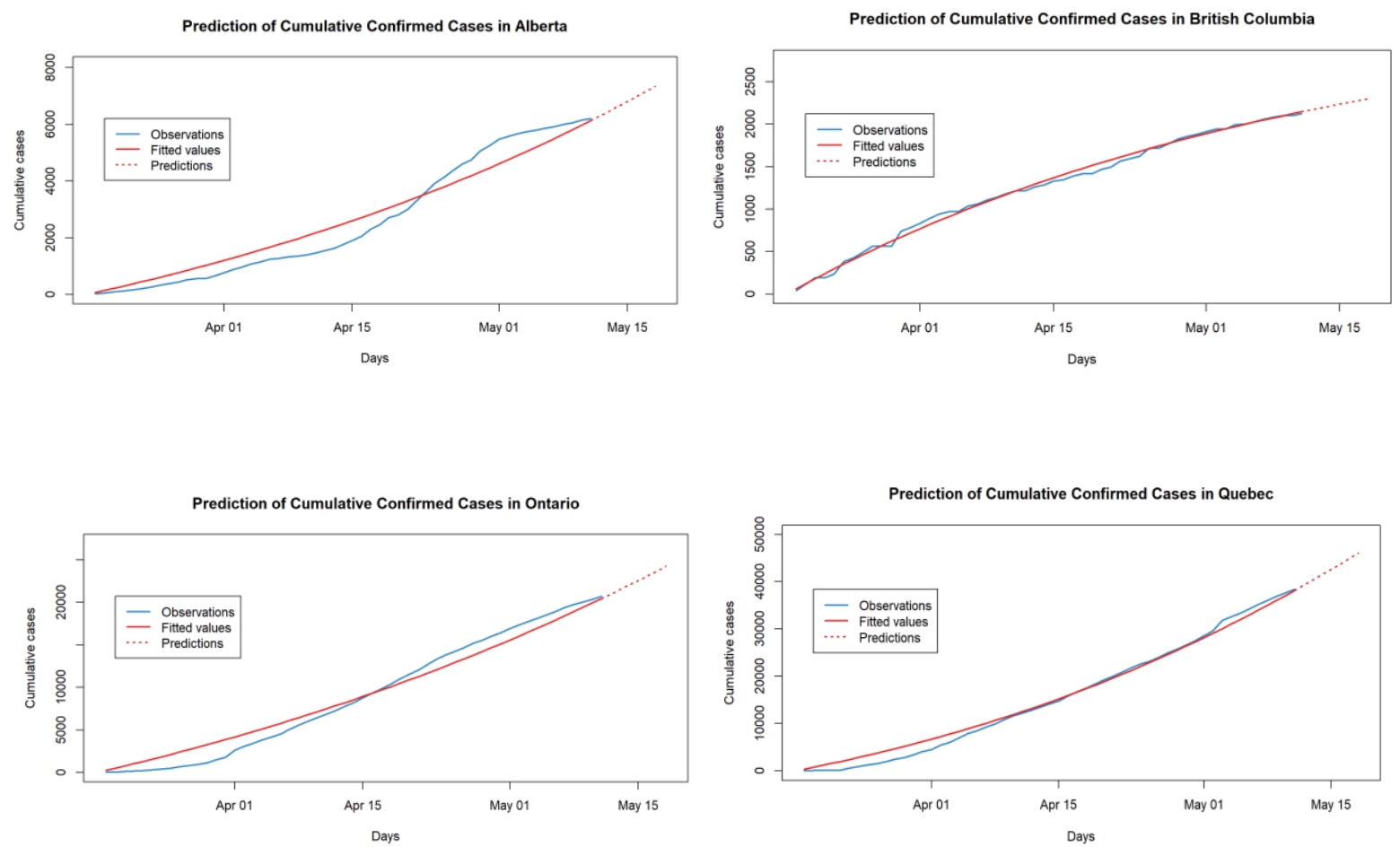

Figure 6. Predicted cumulative cases in Alberta, British Columbia, Ontario, Quebec by SIR model
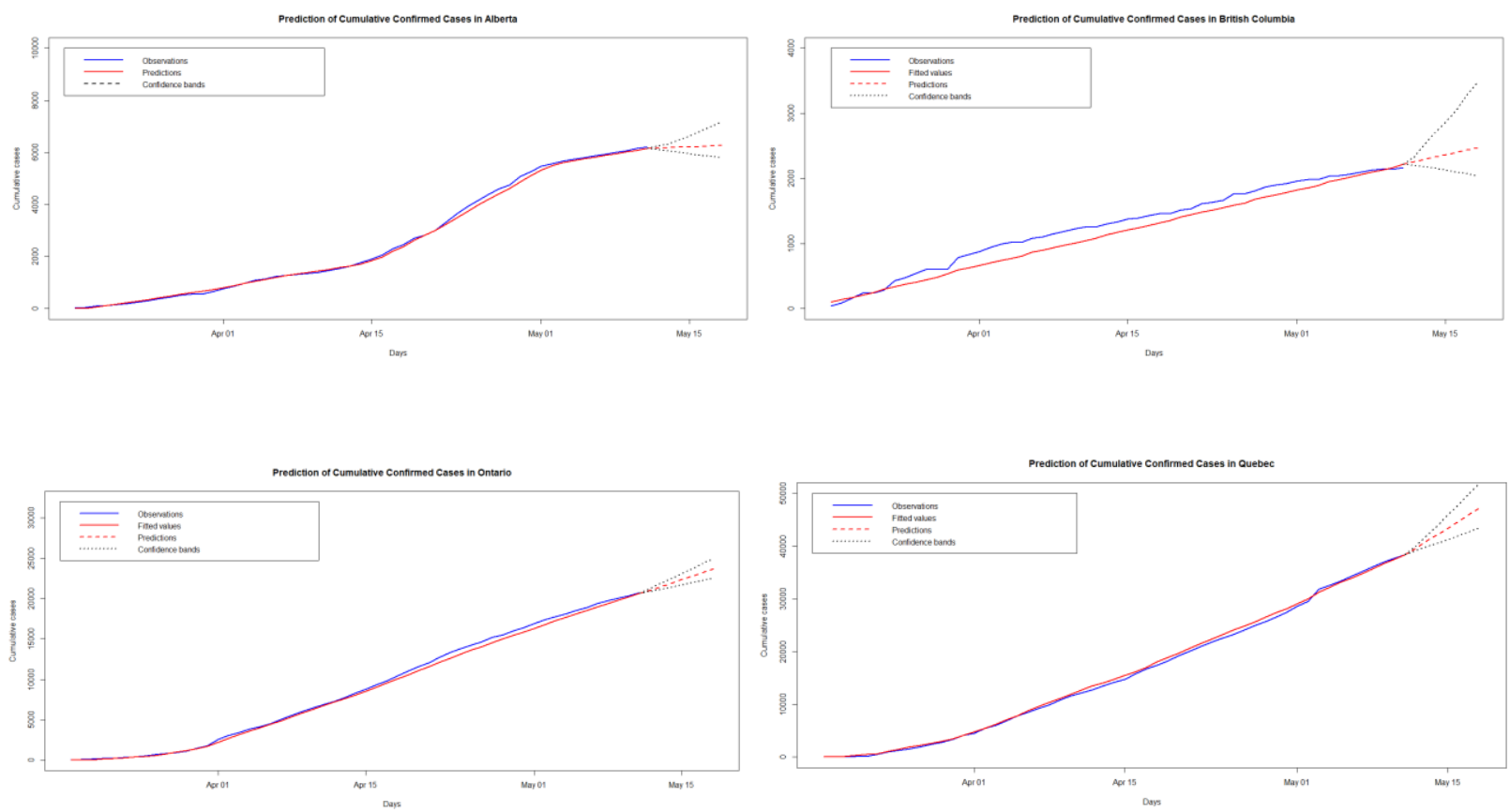

Figure 7. Predicted cumulative cases in Alberta, British Columbia, Ontario, Quebec by NN

We conclude that predictive results largely depend on its model assumptions and it is difficult to find a universal model that reflects and predicts the future trends well for all provinces in Canada. Due to the different situations and limited data availability across provinces, we would suggest choosing the predictive models that best describe the current trends and acquires predictive cases from the models. 


\section{Research Manuscripts}

RESEARCH MANUSCRIPTS is a section to publish cutting-edge research methods and results on the COVID-19 data from our group. We hope to use this site to share our research on COVID-19 with the public in a timely fashion. Currently, we offer recommendations from statistical viewpoints for clinicians and physicians to make reasonable evaluations and resource allocation for the urgent lab test requests from the public. In addition, given the systematic error from case reporting due to multiple reasons, we conducted meta-analysis and sensitivity study to estimate basic reproduction number, average incubation time, asymptomatic infection rate and case fatality rate for COVID-19 to measure the severity of the virus spread. These two pre-printed papers are currently available on the website.

\section{Others}

Our platform collects available public data from multiple reliable sources and can be found in PUBLIC DATA SOURCES. The majority are from the Government of Canada, the provincial government in Canada (https://www.canada.ca/en/public-health/services/diseases/2019-novel-coronavirus-infection.html?topic=tilelink), World Health Organization (https://www.who.int/emergencies/diseases/novel-coronavirus-2019), John Hopkins University (Dong, Du \& Gardner, 2020), and Esri Canada, in which our visualized graphs and statistical analyses are mainly based upon. We realize the importance of more available data for the analysis and hope to explore and access more data so we can provide a more comprehensive picture of the impact of COVID-19 on Canada. This section offers quick access to a collection of data sources for researchers and industry professionals to conduct their own research and industrial work on COVID-19.

The purpose of SELECTED NEWS and STOP VIRUS is to provide news updates and links for self-assessment tools in the local region as well as preventive measures suggested by the government. We also create READER'S CORNER for website visitors to share opinions or feedback.

\section{Discussion and Future Direction}

This paper summarizes a new interactive web-based platform that focuses on the COVID-19 situation in Canada. The main sections on the platform are introduced in detail with existing analysis results presented in both figures and tables to understand the current development of COVID-19 and predict the trends in the near future. We hope the public, healthcare personnel, researchers, and analysts will make use of our website in their decision and policy making. Since COVID-19 is still evolving, we plan to continue to explore different aspects of the impact COVID-19 has imposed and incorporate additional features to the platform. Although web-based platform provides information only for the COVID-19 development in Canada, the analysis code is generic and can be extended to other countries/regions in the world if the similar formatted data are available.

\section{Conflict of Interest}

All the authors have no conflict of interest to declare.

\section{Funding Sources}

The research is partially supported by funding from the Natural Sciences and Engineering Research Council of Canada (NSERC). Yi is Canada Research Chair in Data Science (Tier 1). Her research was undertaken, in part, thanks to funding from the Canada Research Chairs Program.

\section{Ethics approval and consent to participate}

Not applicable.

\section{References}

Chatfield, C., \& Xing, H. (2019). The analysis of time series: an introduction with $R$. CRC press. https://doi.org/10.1201/9781351259446

Dong, E., Du, H., \& Gardner, L. (2020). An interactive web-based dashboard to track COVID-19 in real time. The Lancet infectious diseases, 20(5), 533-534. https://doi.org/10.1016/S1473-3099(20)30120-1

Fanelli, D., \& Piazza, F. (2020). Analysis and forecast of COVID-19 spreading in China, Italy and France. Chaos, Solitons \& Fractals, 134, 109761. https://doi.org/10.1016/j.chaos.2020.109761

Government of Canada. (2020). Coronavirus disease (COVID-19): Outbreak update. Retrieved May 19, 2020 from https://www.canada.ca/en/public-health/services/diseases/2019-novel-coronavirus-infection.html?topic=tilelink

Keeling, M. J., \& Rohani, P. (2011). Modeling infectious diseases in humans and animals. Princeton University Press. https://doi.org/10.2307/j.ctvcm4gk0

Oancea, B., \& Ciucu, Ş. C. (2014). Time series forecasting using neural networks. arXiv preprint arXiv:1401.1333. 
Patterson, J., \& Gibson, A. (2017). Deep learning: A practitioner's approach. " O'Reilly Media, Inc.".

Sievert, C. (2020). Interactive Web-Based Data Visualization with $R$, plotly, and shiny. CRC Press. https://doi.org/10.1201/9780429447273

Wang, C., Liu, L., Hao, X., Guo, H., Wang, Q., Huang, J., ... \& Wei, S. (2020). Evolving epidemiology and impact of non-pharmaceutical interventions on the outbreak of Coronavirus disease 2019 in Wuhan, China. MedRxiv. https://doi.org/10.1101/2020.03.03.20030593

World Health Organization. (2020). Coronavirus disease (COVID-19) pandemic. Retrieved May 19, 2020, from https://www.who.int/emergencies/diseases/novel-coronavirus-2019

\section{Copyrights}

Copyright for this article is retained by the author(s), with first publication rights granted to the journal.

This is an open-access article distributed under the terms and conditions of the Creative Commons Attribution license (http://creativecommons.org/licenses/by/4.0/). 